\title{
Psicooncología
}

ISSN: $1696-7240$

http://dx.doi.org/10.5209/PSIC.57081

\section{Distrés emocional y problemas asociados en población oncológica adulta}

\author{
Paula Martínez López ${ }^{1, *}$; Yolanda Andreu Vaillo²; María José Galdón Garrido ${ }^{3}$; Rocío Romero \\ Retes $^{4}$; Ana García-Conde Benet ${ }^{5}$; Pilar Llombart Fuertes ${ }^{6}$
}

Recibido: 30 de julio de 2017 / Aceptado: 25 de agosto de 2017

Resumen. Objetivo: en este estudio se estima la prevalencia de distrés, se identifican los problemas asociados y se analiza el conocimiento y uso que hacen los pacientes sobre los recursos psicosociales disponibles en el centro hospitalario. Método: se lleva a cabo un estudio transversal en el que participan 203 pacientes oncológicos que están recibiendo tratamiento quimioterápico. En la evaluación se emplean el Inventario de Síntomas Breve-18 (BSI-18), el Listado de Problemas de NCCN y una encuesta sobre el conocimiento y uso de los recursos asistenciales del centro hospitalario. Resultados: la prevalencia de distrés en la muestra total es del $26 \%$. Los problemas físicos son la principal fuente de distrés: fatiga, dolor, sequedad o picor en la piel y problemas de sueño, entre los más prevalentes. El BSI-18 y la Lista de Problemas muestran correlaciones estadísticamente significativas $(r=0,74$; Rango: $r=0,69$ y $\mathrm{r}=0,18$ ). Sólo el $21 \%$ de los casos clínicos de distrés utiliza los servicios psicosociales disponibles. El $79 \%$ restante indica no hacer uso de los mismos por considerar no necesitarlos o por desconocer su existencia. Conclusiones: aunque la mayor parte de los participantes muestran un buen ajuste a la enfermedad, un porcentaje significativo presenta niveles clínicos de distrés. Es crucial llevar a cabo cribados rutinarios de la respuesta de distrés para su manejo/control temprano, e informar a la población sobre los recursos psicosociales disponibles, concienciándolos de la importancia de su uso en momentos de vulnerabilidad. Palabras clave: distrés emocional, BSI-18, Lista de Problemas NCCN, cribado, cáncer.

\section{[en] Emotional distress and problems associated with adult oncological population}

Abstract. Objective: this study estimates the prevalence of emotional distress, identifies the associated problems, and analyses the knowledge and use that patients make of the psychosocial resources available in the Hospital. Method: a cross-sectional study is carried out involving 203 cancer patients receiving

1 Unidad de Investigación "Personalidad y Salud". Universidad de Valencia.

E-mail: paula.martinez@uv.es

2 Unidad de Investigación "Personalidad y Salud". Universidad de Valencia.

E-mail: yolanda.andreu@uv.es

3 Unidad de Investigación "Personalidad y Salud". Universidad de Valencia.

E-mail: maria.j.galdon@uv.es

4 Unidad de Psicología. Fundación Instituto Valenciano de Oncología.

E-mail: rromero@fivo.org

5 Unidad de Psicología. Fundación Instituto Valenciano de Oncología.

E-mail: ana.garcia-conde@uv.es

6 Unidad de Psicología. Fundación Instituto Valenciano de Oncología.

E-mail: pllombart@correo.cop.es

* Dirección de correspondencia: Paula Martínez López. Departamento de Personalidad, Evaluación y Tratamientos Psicológicos. Facultad de Psicología. Universidad de Valencia. Avenida Blasco Ibáñez, 21. CP. 46010. Email: paula.martinez@uv.es 
chemotherapy. The Brief Inventory of Symptoms -18 (BSI 18), the NCCN Problem List, and a shorter version of the Psychosocial Questionnaire are used. Results: the prevalence of distress in the total sample was $26 \%$. The physical problems are the main source of distress: fatigue, pain, skin dry/itchy, and sleep related problems, are the most prevalent. The BSI-18 and the List of Problems show statistically significant correlations $(r=.74$, Range: $r=.69$ and $r=.18)$. Only $21 \%$ of the distress clinical cases use the available psychosocial services. The remaining $79 \%$ indicate that they do not use them because they not need any help or not know about their existence. Conclusions: although the majority of the participants have a good adjustment to the disease, a significant percentage presents clinical levels of emotional distress. It is crucial to carry out routine screening of the distress response for early management/control, and to inform the population about the availability of psychosocial resources, making them aware of the importance of their use in vulnerability moments.

Keywords: emotional distress; BSI-18; NCCN Problem List; screening; cancer.

Sumario. 1. Introducción 2. Método 3. Resultados 4. Discusión 5. Agradecimientos 6. Referencias bibliográficas.

Cómo citar: Martínez López P, Andreu Vaillo Y, Galdón Garrido MJ, Romero Retes R, García-Conde Benet A, Llombart Fuertes P. Distrés emocional y problemas asociados en población oncológica adulta. Psicooncología 2017;14:217-228. Doi: 10.5209/PSIC.57081.

\section{Introducción}

Recientemente, en febrero de 2017, los miembros de la Acción Conjunta para el Control del Cáncer (CanCon), en el marco del Programa de Salud de la Unión Europea, han dado a conocer la Guía Europea para la Mejora de la Calidad en el Control Integral del Cáncer (European Guide on Quality Improvement in Comprehensive Cancer Control) (1). Entre las distintas recomendaciones que se recogen en el documento, destaca la necesidad de realizar un cribado rutinario tanto del nivel de distrés emocional como de las necesidades psicosociales de los pacientes a lo largo de todo el proceso de la enfermedad, con la finalidad de proporcionar una atención especializada, si se requiere $^{(1)}$. Una propuesta sobre la que se lleva trabajando y se insiste desde hace ya más de una década ${ }^{(2-4)}$. En efecto, la agencia de salud pública del Gobierno Federal de Canadá (Canadian Estrategy for Cancer Control) aprobó en 2004 la declaración del distrés emocional como el " 6 " signo vital" en los pacientes de cáncer ${ }^{(5)}$. Declaración que implica la necesidad de controlar sistemáticamente la respuesta de distrés emocional, al igual que el resto de sus precedentes (pulso, respiración, presión sanguínea, temperatura y dolor $)^{(6)}$. Posteriormente, esta propuesta fue respaldada por la Sociedad Internacional de Psico-Oncología (IPOS) durante el Congreso Internacional que dicho organismo celebró en 2009, y también por la Unión Internacional Contra el Cáncer (UICC) durante el Congreso Mundial de Medicina que tuvo lugar en $2010^{(7)}$.

Según la NCCN, la respuesta de distrés emocional se definiría como "una experiencia emocional desagradable y multifactorial de naturaleza psicológica, social y/o espiritual que puede interferir en la capacidad de afrontar de forma efectiva el cáncer, los síntomas físicos asociados y el tratamiento pautado" ${ }^{(8)}$. Así, este término incluiría una variedad de respuestas que se extenderían a lo largo de un continuo, abarcando desde reacciones emocionales "normales" y frecuentes a la enfermedad (vulnerabilidad, tristeza, temor, etc.) hasta aquellas otras más "severas" o "graves" que pueden generar disfuncionalidad en el paciente (cuadros clínicos de ansiedad, 
pánico, depresión; crisis existenciales y/o espirituales, etc.). Con respecto a este extremo del continuo, un meta-análisis sobre prevalencia de distrés en población oncológica adulta señala un porcentaje que oscila entre el 30\% y el 40\% ${ }^{(9,10)}$. Así pues, aproximadamente cuatro de cada diez pacientes de cáncer muestran niveles de distrés clínico en algún momento del proceso de la enfermedad. Esta respuesta emocional parece estar modulada por distintos factores, entre los que se encuentran aquellos relacionados con la enfermedad. Así, diversos estudios informan de variaciones en las tasas de prevalencia de distrés según, por ejemplo, la localización del tumor, la fase del proceso asistencial o el tipo de tratamiento recibido. Zabora et al. en un estudio en el que participan cerca de 4.500 pacientes, señalan un porcentaje medio de distrés clínico del $35 \%$, con un rango que, según la localización del tumor, se sitúa entre el $43 \%$ y el $30 \%{ }^{(11)}$. Los porcentajes más elevados corresponderían al grupo de pacientes con cáncer de pulmón seguidos por aquellos con tumor cerebral, cáncer de hígado y páncreas; en el extremo opuesto del intervalo de distrés, se situaría el grupo de pacientes con cáncer ginecológico. Carlson et al. encuentran resultados similares en una muestra de casi 2.800 pacientes: una prevalencia de distrés clínicamente significativo del $58 \%$ en cáncer de pulmón y porcentajes cercanos al $30 \%$ en cáncer ginecológico, mama, melanoma, colon y próstata ${ }^{(12)}$. Por último, Linden et al. informan de diferencias estadísticamente significativas en distrés según la localización de tumor, correspondiendo las tasas más altas a los pacientes con cáncer de pulmón o hematológico ${ }^{(13)}$. En líneas generales, los resultados de éstos y otros estudios señalan que los diagnósticos asociados a un peor pronóstico conllevan un mayor riesgo de morbilidad psicológica. Finalmente, y aunque en este caso los resultados no son concluyentes, algunos estudios señalan como periodos de máxima vulnerabilidad psicológica los momentos temporales cercanos al diagnóstico ${ }^{(14,15)} \mathrm{y}$ al tratamiento activo de la enfermedad ${ }^{(16)}$.

Desafortunadamente, menos de la mitad de los casos clínicos de distrés se identifican y derivan a los servicios de asistencia psicosocial correspondientes ${ }^{(17)}$, con las repercusiones que todo ello conlleva en cuanto a disminución en la calidad de vida del paciente y aumento del gasto en la asistencia sanitaria recibida ${ }^{(18)}$; no sólo por la probabilidad de realizar un mayor número de pruebas diagnósticas y pautar tratamientos médicos innecesarios, sino también por la peor adherencia del paciente a las recomendaciones médicas.

En este contexto, se desarrolla el presente estudio, cuyo objetivo es estimar la prevalencia de distrés clínicamente significativo en una muestra heterogénea de pacientes oncológicos adultos. Asimismo, se explora la problemática existente más habitual y se analiza su posible relación con los niveles de distrés. Finalmente, se estudia el conocimiento que tienen los pacientes sobre los recursos de asistencia psicosocial ofrecidos por el centro oncológico, su nivel de utilización y la posible discrepancia entre dicho uso y el porcentaje de casos clínicos de distrés identificados.

\section{Método}

\subsection{Procedimiento y participantes}

Este trabajo forma parte de un proyecto de investigación más amplio sobre detección $\mathrm{y}$ tratamiento del distrés emocional en pacientes oncológicos adultos y fue aprobado 
por el Comité Ético de Investigación Clínica de la Fundación Instituto Valenciano de Oncología (FIVO). Entre septiembre y noviembre de 2016, los pacientes que acudían al Hospital de Día del FIVO para recibir tratamiento de quimioterapia eran invitados a participar. Los criterios de inclusión fueron: ser mayor de 18 años, estar diagnosticado de cáncer y tener capacidad para dar el consentimiento informado. Los pacientes eran informados sobre el objeto del estudio y, aquellos que mostraron interés por participar en el mismo, dieron su consentimiento informado. Del total de pacientes aproximados $(\mathrm{N}=215)$, sólo un $6 \%$ rechazó participar. Así, la muestra final estuvo compuesta por 203 pacientes oncológicos.

\subsection{Instrumentos}

- Los datos sociodemográficos se recogieron mediante una hoja de datos que completaban los participantes. En concreto, se les solicitaba que informaran sobre su edad, su estado civil, su nivel educativo y su situación laboral actual.

- Los datos médicos se obtuvieron mediante la consulta de las historias clínicas. Entre las variables médicas revisadas se contemplaron la localización de tumor, el estadio de la enfermedad y el tipo de tratamiento quimioterapéutico (ya fuera de primera línea o por recaída).

- Inventario de Síntomas Breve-18 (BSI-18) ${ }^{(19)}$. Autoinforme compuesto por 18 ítems, medidos en una escala Likert de 5 puntos, que evalúa el grado de distrés o malestar emocional que ha experimentado una persona durante la última semana. El instrumento ofrece puntuaciones en las tres subescalas que lo conforman (somatización, depresión y ansiedad) y una puntuación total en distrés (Global Severity Index; GSI). Este instrumento permite también la identificación de un caso clínico de distrés a partir del criterio que sugiere el autor: $\mathrm{T} \geq 63$ en la puntuación total de la escala o, en al menos, dos de las subescalas que lo conforman. El BSI-18 ha mostrado propiedades psicométricas satisfactorias en población oncológica española ${ }^{(20,21)}$. En la muestra de estudio, el BSI-18 mostró una satisfactoria consistencia interna $\left(\alpha_{\mathrm{GSI}}=0,93 ; \alpha_{\text {somatización }}=0,75 ; \alpha_{\text {depresión }}=0,86 ; \alpha_{\text {ansiedad }}=0,86\right)$.

- Listado de Problemas de la $\mathrm{NCCN}^{(8)}$. Lista estandarizada de dificultades que la NCCN propone para identificar el origen del malestar emocional según cinco áreas diferentes: problemas prácticos (5 ítems), problemas familiares (3 ítems), problemas emocionales (6 ítems), problemas físicos (21 ítems) e inquietudes espirituales o religiosas (1 ítem).

- Cuestionario Psicosocial ${ }^{(12)}$. Esta encuesta breve consta de 6 ítems relativos al nivel de información que posee el paciente sobre los servicios de asistencia psicosocial existentes en el centro hospitalario y al grado de utilización de los mismos.

\subsection{Análisis estadísticos}

Los análisis fueron realizados utilizando el paquete estadístico SPSS para Windows (versión 22.0). Se calcularon estadísticos descriptivos para (i) obtener los datos sociodemográficos, médicos y psicológicos que definen la muestra de estudio; (ii) estimar la prevalencia de distrés emocional en la muestra; (iii) describir los problemas más prevalentes según la lista de problemas e (iv) identificar el grado de 
conocimiento y uso que hace la muestra de los recursos psicosociales disponibles. Se calcularon correlaciones de Pearson para explorar la posible relación entre los niveles de distrés y la problemática identificada. El nivel de significación estadística utilizado fue 0,05 .

\section{Resultados}

La muestra final de este estudio la componían 203 pacientes oncológicos de entre 25 y 82 años de edad, con una media de 53 años $(\mathrm{SD}=12,11)$. El 76\% $(\mathrm{N}=154)$ eran mujeres y el $24 \%(\mathrm{~N}=49)$ hombres. La mayoría de los participantes estaban casados o vivían en pareja $(73 \% ; \mathrm{N}=149)$, tenían al menos estudios primarios $(86 \% ; \mathrm{N}=$ 173) y, en el momento de la evaluación, se encontraban en situación de inactividad laboral ( $61 \% ; \mathrm{N}=123)$, ya fuera por baja laboral (31\%) o como pensionistas/jubilados (30\%). Respecto a las características médicas, la localización tumoral más frecuente fue cáncer de mama ( $47 \% ; \mathrm{N}=96)$, seguido a distancia por cáncer genital femenino $(16 \% ; \mathrm{N}=32)$, del aparato digestivo $(12 \% ; \mathrm{N}=24)$ y del tracto respiratorio $(10 \%$; $\mathrm{N}=20)$. El $66 \%(\mathrm{~N}=134)$ de los participantes recibía tratamiento quimioterápico por un tumor primario y el $29 \%(\mathrm{~N}=58)$ por una recaída. Los datos de las variables sociodemográficas y médicas se encuentran recogidos en la tabla 1.

Tabla 1. Perfil sociodemográfico y médico de la muestra $(\mathrm{N}=203)$

\begin{tabular}{lcc}
\hline & $N$ & $\%$ \\
\hline Edad $(M ; S D):$ & 53,24 & 12,11 \\
Sexo: & & \\
$\quad$ Mujer & 154 & 75,9 \\
$\quad$ Hombre & 49 & 24,1 \\
\hline Convivencia: & & \\
$\quad$ Casado/en pareja & 149 & 73,4 \\
$\quad$ Soltero/separado/viudo & 54 & 26,6 \\
\hline Nivel educativo (N=199): & & \\
$\quad$ Sin estudios & 24 & 11,8 \\
$\quad$ Estudios primarios & 92 & 45,3 \\
$\quad$ Estudios secundarios & 42 & 20,7 \\
$\quad$ Estudios superiores & 41 & 20,2 \\
\hline Situación laboral: & & \\
En activo & 18 & 8,9 \\
Tareas del hogar & 45 & 22,2 \\
Baja laboral & 63 & 31,0 \\
Jubilado & 60 & 29,6 \\
Desempleado & 16 & 7,9 \\
\hline
\end{tabular}




\begin{tabular}{lcc}
\hline Localización de tumor: & & \\
Mama & 96 & 47,3 \\
Ginecológico & 32 & 15,8 \\
Gastro-intestinal & 24 & 11,8 \\
Tracto respiratorio & 20 & 9,9 \\
Aparato urinario & 12 & 5,9 \\
Órganos hematopoyéticos & 8 & 3,9 \\
Otros & 11 & 5,4 \\
\hline Estadio de la enfermedad: & & \\
0 & 4 & 2,0 \\
I & 14 & 6,9 \\
II & 51 & 25,1 \\
III & 47 & 23,2 \\
IV & 87 & 42,9 \\
\hline Fase proceso asistencial $(N=192):$ & & \\
Tratamiento tumor primario & 134 & 66,0 \\
Tratamiento por recaida & 58 & 28,6 \\
\hline
\end{tabular}

\subsection{Niveles y prevalencia de distrés}

Se obtuvo un valor medio en GSI de 12,11 ( $\mathrm{SD}=12,02$; rango: 0-64). Por subescalas, las puntuaciones medias en las subescalas fueron muy similares: ansiedad $(\mathrm{M}=$ $4,07 ; \mathrm{SD}=4,71$; rango: $0-22)$, somatización $(\mathrm{M}=4,06 ; \mathrm{SD}=3,99$; rango: $0-21)$ y depresión ( $\mathrm{M}=3,98 ; \mathrm{SD}=4,67$; rango: $0-22)$. Respecto a la prevalencia de distrés clínico, y siguiendo el criterio propuesto por Derogatis ${ }^{(19)}$ para la identificación de un "caso clínico", el 26\% de la muestra (53 de 203) presentó niveles clínicamente significativos de distrés. De forma particular, en el subgrupo de pacientes con cáncer de mama (aquel con un tamaño muestral mayor) se alcanzó un porcentaje de distrés del 35\%, es decir, 34 de las 96 mujeres que conformaban el grupo mostraron niveles clínicamente significativos de distrés.

\subsection{Fuentes de distrés psicológico}

Tal y como se observa en la Tabla 2, los participantes señalaron un total de 1.769 problemas como posible origen de su distrés. Siguiendo la agrupación propuesta por la $\mathrm{NCCN}^{(8)}$, las categorías más frecuentemente señaladas por la muestra total de estudio fueron los problemas físicos, que representaban más de la mitad (62\%; $\mathrm{N}=1.099$ ) del total de problemas registrados y los problemas emocionales que suponían algo más de uno de cada cuatro de los problemas informados $(28 \%$, $\mathrm{N}=489$ ). El resto de categorías registraron porcentajes considerablemente más bajos (por orden): problemas prácticos $(4 \% ; \mathrm{N}=71)$, problemas familiares $(4 \%$; $\mathrm{N}=70)$ e inquietudes religiosas o espirituales $(2 \% ; \mathrm{N}=29)$. El subgrupo "cáncer 
de mama" $(\mathrm{N}=96)$ obtuvo una distribución similar a la registrada por la muestra total: problemas físicos $(60 \% ; \mathrm{N}=534)$; problemas emocionales $(30 \% ; \mathrm{N}=265)$; problemas prácticos $(4 \% ; \mathrm{N}=39)$; problemas familiares $(4 \% ; \mathrm{N}=38)$ e inquietudes religiosas o espirituales $(2 \% ; \mathrm{N}=13)$.

Tabla 2. Descriptivos sobre las fuentes de distrés $(N=203)$

\begin{tabular}{|c|c|c|c|c|c|}
\hline & $\%$ & $N$ & & $\%$ & $N$ \\
\hline Problemas Emocionales & & 489 & Problemas Físicos & & 1.099 \\
\hline Preocupación & 23,51 & 115 & Dolor & 8,19 & 90 \\
\hline Temor & 19,63 & 96 & Náuseas & 3,18 & 35 \\
\hline Tristeza & 18,40 & 90 & Cansancio o fatiga & 11,56 & 127 \\
\hline Depresión & 6,34 & 31 & Sueño & 7,28 & 80 \\
\hline Nerviosismo & 22,09 & 108 & Problemas movilidad & 4,91 & 54 \\
\hline \multirow[t]{2}{*}{ Anhedonia } & 10,02 & 49 & Dificultad bañarse / vestirse & 2,09 & 23 \\
\hline & & & Respiración & 3,00 & 33 \\
\hline Problemas Familiares & & 70 & Llagas bucales & 4,00 & 44 \\
\hline con la pareja & 37,14 & 26 & Alimentación & 2,64 & 29 \\
\hline con los hijos & 34,29 & 24 & Indigestión & 1,36 & 15 \\
\hline \multirow[t]{2}{*}{ con otros familiares } & 28,57 & 20 & Estreñimiento & 6,92 & 76 \\
\hline & & & Diarrea & 2,73 & 30 \\
\hline Problemas prácticos & & 71 & Cambios en hábitos de orinar & 3,28 & 36 \\
\hline Vivienda & 21,13 & 15 & Fiebre & 1,36 & 15 \\
\hline Seguro/económicos & 8,45 & 6 & Sequedad/picores de piel & 7,73 & 85 \\
\hline Trabajo/escuela & 25,35 & 18 & Sequedad/ congestión nasal & 4,55 & 50 \\
\hline Transporte & 18,31 & 13 & Cosquilleo en las manos/pies & 5,46 & 60 \\
\hline \multirow[t]{2}{*}{ Cuidado de niños/mayores } & 26,76 & 19 & Sensación de hinchazón & 5,28 & 58 \\
\hline & & & Sexuales & 3,82 & 42 \\
\hline \multirow[t]{2}{*}{ Religiosos/espirituales } & & 29 & Aspecto físico & 4,91 & 54 \\
\hline & & & Memoria/concentración & 5,73 & 63 \\
\hline Otros problemas & & 11 & Total problemas & & 1,769 \\
\hline
\end{tabular}

Un análisis pormenorizado de las diferentes categorías de problemas en la muestra total de estudio indicaba que, entre los problemas físicos $(\mathrm{N}=1.099)$, la fatiga ( $12 \%$; $\mathrm{N}=1.127)$, el dolor $(8 \% ; \mathrm{N}=90)$, los problemas de sequedad y/o picores en la piel $(8 \% ; \mathrm{N}=85)$ y aquellos relacionados con el sueño $(7 \% ; \mathrm{N}=80)$ eran los más frecuentemente señalados (ver Tabla 2). Respecto a los problemas emocionales $(\mathrm{N}=489)$, la sintomatología ansiosa fue la más prevalente (por orden): preocupación $(24 \% ; \mathrm{N}=115)$, nerviosismo $(22 \% ; \mathrm{N}=108)$ y temor $(20 \%$; $\mathrm{N}=96$ ); mientras que la sintomatología depresiva obtenía frecuencias más bajas: tristeza $(18 \% ; \mathrm{N}=90)$, depresión $(6 \% ; \mathrm{N}=31)$ y anhedonia $(10 \% ; \mathrm{N}=49)$. Con respecto a los problemas prácticos $(\mathrm{N}=71)$, los problemas relacionados con el cuidado de un tercero (independientemente de su edad) $(27 \% ; \mathrm{N}=19)$ eran los más 
señalados por los participantes, aunque seguidos muy de cerca por la presencia de problemas en el trabajo y/o con los estudios $(25 \% ; \mathrm{N}=18)$. Por último, en cuanto a los problemas familiares $(\mathrm{N}=70)$, destacan los problemas de relación de pareja $(37 \% ; \mathrm{N}=26)$ (ver Tabla 2$)$.

\subsection{Relación entre niveles de distrés y problemas informados}

En la Tabla 3 se presentan datos sobre la asociación entre la respuesta de distrés y las categorías de problemas que componen el Listado de problemas de la NCCN. Como se puede observar, la respuesta de distrés mostraba una correlación estadísticamente significativa con la presencia de dificultades informadas por el paciente. Así, niveles elevados de distrés en la muestra total se relacionaron con mayor número de dificultades en general, $(\mathrm{r}=0,57 ; \mathrm{p} \leq 0,001)$, siendo la relación entre distrés y problemas emocionales $(\mathrm{r}=0,56 ; \mathrm{p} \leq 0,001)$ la más elevada. Asimismo, mayores niveles de distrés se relacionaron con un mayor número de problemas físicos $(\mathrm{r}=0,44 ; \mathrm{p} \leq 0,001)$, problemas familiares $(\mathrm{r}=0,37 ; \mathrm{p} \leq 0,001)$ y problemas prácticos $(\mathrm{r}=0,27 ; \mathrm{p} \leq 0,001)$. La relación entre distrés e inquietudes religiosas y/o espirituales, aunque significativa, fue la más débil $(\mathrm{r}=0,18 ; \mathrm{p}=0,011)$. En el subgrupo de cáncer de mama, los resultados nuevamente se replicaron (ver tabla 3 ).

Tabla 3. Relación entre distrés y fuentes de problemas.

\begin{tabular}{lcccc}
\hline & \multicolumn{2}{c}{ Muestra total $(N=203)$} & \multicolumn{2}{c}{ Subgrupo mama $(N=96)$} \\
\cline { 2 - 5 } & GSI & caso/no caso & GSI & caso/no caso \\
\hline Problemas prácticos &, $435^{* * *}$ &, $266^{* * *}$ &, $457 * * *$ &, $291 * *$ \\
Problemas familiares &, $478^{* * *}$ &, $370^{* * *}$ &, $573 * * *$ &, $419 * * *$ \\
Problemas emocionales &, $668^{* * *}$ &, $558^{* * *}$ &, $692 * * *$ &, $573 * * *$ \\
Inquietudes religiosas/espirituales &, $180^{*}$ &, $181^{*}$ &, $283 * *$ &, $224 *$ \\
Problemas físicos &, $587 * * *$ &, $441 * * *$ &, $592 * * *$ &, $461 * * *$ \\
Total problemas &, $741^{* * *}$ &, $571 * * *$ &, $754 * * *$ &, $586 * * *$ \\
\hline
\end{tabular}

${ }^{*} p<0,05 . * * p<0,01 ; * * * p<0,001$

\subsection{Conocimiento y uso de los servicios de asistencia psicosocial}

Los resultados obtenidos indicaron que el $84 \%(\mathrm{~N}=171)$ de la muestra total conocía la existencia del servicio de psico-oncología con el que cuenta el centro hospitalario. De hecho, 65 pacientes informaban de su asistencia al mismo, bien en el pasado $(\mathrm{N}=44)$ bien en el momento del estudio $(\mathrm{N}=21)$ para recibir en su mayoría atención individual (94\%). Los usuarios del servicio eran principalmente los propios pacientes $(88 \%) \mathrm{y}$, en mucha menor proporción, sus parejas $(18 \%)$ $\mathrm{u}$ otros miembros de la familia (6\%). Por el contrario, entre las razones que manifestaban los pacientes para no utilizar los recursos psicosociales disponibles destacó, sobre todo, la consideración de no necesitarlo $(76 \%)$ y, en mucha menor medida, su desconocimiento sobre la existencia del mismo (14\%). Atendiendo de forma exclusiva a los pacientes con niveles clínicamente significativos de distrés, 
cabe señalar que un porcentaje reducido (21\%) informó de la utilización actual de dichos recursos, mientras que un $79 \%$ indicó no hacer uso de ellos. Las razones para no acudir al servicio de psicología del centro seguían una distribución similar a la descrita para la muestra total.

\section{Discusión}

Uno de los objetivos de este estudio fue estimar la prevalencia de distrés y conocer su origen en una muestra de 203 pacientes oncológicos adultos que estaban recibiendo tratamiento quimioterápico. Al respecto, nuestros resultados señalan que la mayor parte de los pacientes oncológicos (74\%) muestra un buen ajuste a la enfermedad en el momento de la evaluación. No obstante, aproximadamente uno de cada cuatro (26\%) presenta niveles clínicos de distrés y en el subgrupo de mujeres diagnosticadas de cáncer de mama, esta prevalencia asciende a una de cada tres (35\%). Nuestros resultados muestran niveles globales de distrés ligeramente inferiores a aquellos recogidos en la literatura ${ }^{(9)}$ y coincidentes con los valores encontrados en otros estudios sobre prevalencia de distrés por localización de tumor. Zabora et al., y Carlson et al., informan de un porcentaje de distrés clínico en el subrupo "mama" del $33 \%$ y del $35 \%$, respectivamente ${ }^{(11,12)}$.

En segundo lugar y con respecto al origen del distrés, nuestros resultados señalan que, en la muestra de estudio, los problemas más frecuentes son los de tipo físico (62\%), destacando fundamentalmente la fatiga, el dolor, la sequedad en la piel y los problemas de sueño. Síntomas todos ellos susceptibles de ser asociados al tratamiento de quimioterapia que están recibiendo los pacientes en el momento de la evaluación. Con la finalización de dicho tratamiento, este tipo de problemas tienden a desaparecer gradualmente y, consecuentemente, se esperaría una reducción en los niveles de distrés. Asimismo, los problemas emocionales surgen como la segunda fuente de distrés. En el $60 \%$ de los casos, la respuesta de distrés se asocia a problemas emocionales de tipo ansioso - preocupación, nerviosismo y temor- y en el $40 \%$ restante, a problemas emocionales asociados a síntomas depresivos -tristeza y pérdida de interés en actividades de la vida cotidiana. Respecto a este tipo de problemas, cabe destacar, además, la estrecha relación que mantienen con los niveles de distrés $(\mathrm{r}=0,67)$. Efectivamente, en su asociación con las diferentes fuentes de distrés, el BSI-18 muestra la correlación más alta con los problemas de origen emocional, subrayando la necesidad de atender -controlar y manejar- esta sintomatología de cara a reducir la presencia de distrés. No obstante, la respuesta de distrés correlaciona significativamente también con el resto de las categorías de dificultades que contempla el Listado de Problemas de la NCCN. Un patrón que, con valores ligeramente superiores, también se mantiene en el subgrupo de cáncer de mama. Así pues, es imprescindible ofrecer una atención integral al paciente en la que, junto a aquellas necesidades directamente derivadas del tratamiento médico de la enfermedad, se aborden también aquellas otras de carácter emocional, social, práctico y espiritual que permitan aliviar su sufrimiento en la experiencia con el diagnóstico y tratamiento del cáncer.

Otro de los objetivos de este estudio fue explorar en la muestra de estudio la posible discrepancia, recogida en la literatura ${ }^{(12,17)}$, entre la presencia de niveles clínicos de distrés y el uso que los pacientes hacen de los recursos psicosociales 
disponibles. Los resultados señalaron que sólo el $21 \%$ de los pacientes con distrés clínico utilizan los recursos psicosociales disponibles, mientras que el $79 \%$ restante no lo hace. Entre los principales motivos indicados por los participantes en el estudio destaca, sobre todo, la consideración de no necesitarlo: aunque también se explicitó el desconocimiento sobre la existencia de los mismos. Quizá -y además de no ser consciente de los recursos de que dispone-, el hecho de que el paciente sea reacio a hablar de sus problemas emocionales con el profesional sanitario al considerar que sólo deben centrarse en el tratamiento médico, el no ser consciente de la gravedad del propio nivel de distrés, o el miedo al estigma de una enfermedad mental, son posibles factores explicativos de esta situación ${ }^{(22)}$. Si este fuera el caso, sería conveniente ofrecer al paciente información psicoeducativa sobre la respuesta de distrés en cáncer (p.ej., información sobre manifestaciones más habituales, evolución, factores de riesgo, etc.) y sobre los recursos psicosociales con los que cuenta en su entorno, resaltando sobre todo la importancia de recurrir a ellos en momentos de cierta vulnerabilidad.

Este estudio no está exento de limitaciones. Algunas de las más importantes hacen referencia a la muestra de estudio. El hecho de trabajar con una muestra de pacientes oncológicos que están recibiendo tratamiento de quimioterapia en el momento de la evaluación limita la generalización de los resultados. Asimismo, hay que señalar que las localizaciones más prevalentes en la muestra de estudio se asocian al sexo femenino; de hecho, del total de la muestra, casi la mitad de los participantes presentaban un diagnóstico de cáncer de mama (47\%), y un $16 \%$ de cáncer ginecológico. Por otra parte y con respecto al diseño, la transversalidad del estudio no permite obtener datos sobre el curso temporal de la respuesta de distrés. Futuras investigaciones deberían así tener en cuenta las consideraciones previas y plantear diseños que nos permitan conocer la evolución temporal de la respuesta de distrés -identificando posibles trayectorias-; así como determinar la prevalencia de distrés, atendiendo tanto a la localización del tumor como a otras variables que la literatura señala como factores moduladores de la respuesta de distrés (p.ej., edad, sexo, fase del proceso asistencial, etc.). Este tipo de trabajos nos ayudarán a seguir profundizando en este tema.

En resumen, los resultados de este estudio respaldan la necesidad de llevar a cabo una evaluación rutinaria de la respuesta de distrés emocional y de sus causas en la población oncológica, con el fin de poder realizar una detección, control y tratamiento temprano. Evaluación que, tal y como recomiendan las guías clínicas para la atención del paciente oncológico ${ }^{(1,6,23)}$, debe realizarse en las diferentes fases del proceso de la enfermedad -sospecha, diagnóstico, tratamiento, seguimiento, recaída, etc. Así mismo, nuestros datos señalan la importancia de informar a los pacientes sobre los diferentes recursos psicosociales con los que cuenta en su entorno y de concienciar a la población sobre la importancia de su uso llegado el momento.

\section{Agradecimientos}

Esta investigación ha sido financiada por el Ministerio de Economía y Competitividad. Gobierno de España (PSI2010-15355). Las autoras agradecen a todos los participantes y al personal sanitario de la Fundación Instituto Valenciano de Oncología su colaboración en este estudio. 


\section{Referencias bibliográficas}

1. CanCON. European Guide on Quality Improvement in Comprehensive Cancer Control, 2017. En línea. [Acceso el 20 de mayo de 2017]. Disponible en: https://cancercontrol.eu/ archived/uploads/images/Guide/pdf/CanCon_Guide_FINAL_Web.pdf

2. Carlson LE, Waller A, Mitchell AJ. Screening for distress and unmet needs in patients with cancer: review and recommendations. J Clin Oncol 2012;30:1160-77. doi: 10.1200/ JCO.2011.39.5509

3. Jacobsen P. Screening for psychological distress in cancer patients: challenges and opportunities. J Clin Oncol 2007;25:4526-7. doi: 10.1200/JCO.2007.13.1367

4. Jacobsen P. Improving psychosocial care in outpatient oncology settings. J Natl Compr Canc Netw 2010;8:368-70. doi: 10.6004/jnccn.2010.0029

5. Rebalance Focus Action Group. A position paper: screening key indicators in cancer patients: pain as a fifth vital sign and emotional distress as a sixth vital sign. Can Strategy Cancer Control Bull 2004;7(Suppl):4.

6. Bultz B, Holland J. Emotional Distress in patients with cancer: The sixth vital sign. Community Oncol 2006;3:311-34.

7. Bult, B, Johansen C. Screening for distress, the 6th Vital Sign: where are we, and where are we going? Psychooncology 2011;20:569-71. doi: 10.1002/pon.1986

8. National Comprehensive Cancer Network [NCCN]. NCCN Clinical Practice Guidelines in Oncology: Distress Management. En línea. 2017. [Acceso el 18 de junio de 2017]. Disponible en: http://www.nccn.org.

9. Mitchell A, Chan M, Bhatti H, Halton M, Grassi L, Johansen C, et al. Prevalence of depression, anxiety, and adjustment disorder in oncological, hematological and palliative care settings: a meta-analysis of 94 interview-based studies. Lancet 2011;12:160-74. doi: 10.1016/S1470-2045(11)70002-X

10. Grassi L, Travado L, Gil F, Sabato S, Rossi E, the SEPOS Group. Psychosocial morbidity and its correlates in cancer patients of the Mediterranean area: findings from the Southern European Psycho-Oncology Study. J Affect Disord 2004;83:243-8. doi: :10.1016/j. jad.2004.07.004

11. Zabora J, Brintzenhofeszoc K, Curbow B, Hooker C, Piantadosi S. The prevalence of psychosocial distress by cancer site. Psychooncology 2001;Doi. 10.19-28. 10.1002/1099-1611(200101/02)10:1<19::AID-PON501>3.0.CO;2-6

12. Carlson L, Angen M, Cullum J, Goodey E, Koopmans J, Lamont L, et al. High levels of untreated distress and fatigue in cancer patients. Br J Cancer 2004;90:2297-304. doi: $10.1038 /$ sj.bjc. 6601887

13. Linden W, Vodermaier A, Mackenzie R, Greig D. Anxiety and depression after cancer diagnosis: prevalence rates by cancer type, gender, and age. J. Affect Disord 2012; 141:343-51. doi: 10.1016/j.jad.2012.03.025

14. Andreu Y, Galdón MJ, Durá E, Martínez P, Pérez S, Murgui, S. A longitudinal study of psychosocial distress in breast cancer: prevalence and risk factors. Psychol Health 2012;27:72-87. doi: 10.1080/08870446.2010.542814

15. Pérez S, Galdón MJ, Andreu Y, Ibáñez E, Durá E, Conchado A, et al. Posttraumatic stress symptoms in breast cancer patients: temporal evolution, predictors, and mediation. $\mathrm{J}$ Trauma Stress 2014:27:224-31. doi: 10.1002/jts.21901

16. Lauzier S, Maunsell E, Levesque P, Mondor M, Robert J, Robidoux A, et al. Psychological distress and physical health in the year after diagnosis of DCIS or invasive breast cancer. Breast Cancer Res Treat 2010:120:685-91. doi: 10.1007/s10549-009-0477-z 
17. Martínez P, Galdón MJ, Andreu Y, Ibáñez E. The distress Thermometer in Spanish cancer patients: convergent validity and diagnostic accuracy. Support Care Cancer 2013;11:3095102. doi: 10.1007/s00520-013-1883-7

18. Andreu Y, Galdón MJ, Martínez P, Martínez O. Precisión diagnóstica del Termómetro de Distrés en neoplasias hematológicas. Psicooncología 2015:12:237-47. doi: 10.5209/ rev_PSIC.2015.v12.n2-3.51006

19. Derogatis LR. Brief Symptom Inventory (BSI-18). Minneapolis, MN: NCS Pearson, 2013.

20. Galdón MJ, Durá E, Andreu Y, Ferrando M, Murgui S, Pérez S, et al. Psychometric proprieties of the Brief Symptoms Inventory 18 (BSI-18) in a Spanish breast sample, J. Psychosom Res 2008;65:533-9. doi: 10.1016/j.jpsychores.2008.05.009

21. Martínez P, Andreu Y, Galdón MJ [bajo revisión]. Brief Symptom Inventory-18: factor structure and invariance among adult patients with cancer.

22. Mitchell AJ. Screening procedures for psychological distress. En Holland J, Breitbart W, Jacobsen P, Lederberg M, Loscalzo M, McCorkle R, editors. Psycho-Oncology ( $2^{\mathrm{a}}$ ed.) New York: Oxford University Press, 2010.p.389-96.

23. National Institute for Clinical Excellence [NICE] (2004). Guidance on cancer services: Improving supportive and palliative care for adults with cancer. En línea. [Acceso el 1 de junio de 2017]. Disponible en: http://www.nice.org.uk. 